\title{
REABILITAÇÃO TOTAL IMEDIATA MAXILO-MANDIBULAR COM PRÓTESE FIXA SOBRE IMPLANTES COM CONCEITO ALL-ON-FOUR: RELATO DE CASO
}

\section{IMMEDIATE DOUBLE FULL ARCH REHABILITATION WITH IMPLANT SUPPORTED PROTHESIS: A CASE REPORT}

\section{Frederico Martins da Silva}

Graduado em Odontologia pela Universidade Federal Fluminense / RJ

Especialista em Prótese Dentária pela $\mathrm{ABO} / \mathrm{RJ}$

\section{Isabelly de Vasconcellos Pereira}

Graduada em Odontologia pela Universidade Federal Fluminense / RJ

Pós-Graduanda do curso de especialização em Implantodontia da Universidade Federal Fluminense / RJ

\section{Rodrigo Figueiredo de Brito Resende}

Doutor em Odontologia pela Universidade Federal Fluminense / RJ.

Professor de Cirurgia Oral Menor da Faculdade de Odontologia da Universidade Federal Fluminense / RJ.

\section{Felipe Silveira Maximo de Almeida}

Doutor em Ciências pelo Instituto Militar de Engenharia / RJ

Professor da Especialização em Prótese Dentária da Associação Brasileira de Odontologia / RJ

\section{Pâmella Santana Nunes}

Graduanda em Odontologia pela Universidade Federal Fluminense / RJ

Faculdade de Odontologia - Universidade Federal Fluminense / RJ

Niterói

Artigo de Relato de Caso

\section{Frederico Martins da Silva}

Endereço: Rua Joaquim Távora, n76, apto 903, bloco 1.

Telefone: (21) 997956018

E-mail: fredericomartinsdasilva@gmail.com 


\section{RESUMO}

Caracterizada por ser uma das mais documentadas formas de reabilitação de dentição completa sobre implantes, a reabilitação com próteses tipo protocolo híbrida, compreende uma infraestrutura metálica associada a uma sobrestrutura em resina, tendo atualmente cerca de 40 anos de acompanhamento da efetividade da técnica. Com o aumento da expectativa de vida das populações, espera-se o aumento da prevalênia do edentulismo completo. Esse aumento gera uma maior necessidade desse tipo de tratamento restaurador e uma consequente melhora da forma de alcançar esse objetivo. O trabalho em questão, busca relatar um caso de cirurgia e função imediata, maxilo-mandibular, incluindo o planejamento clínico, fotográfico e radiográfico. Foi feita a cirurgia de instalação de 8 implantes, sendo 4 do tipo hexágono externo(HE) no arco superior e 4 hexágono interno(HI) inferior. Foram utilizadas angulações distais nos implantes mais posteriores de ambos arcos, compensados com minipilares angulados, diminuindo o cant-lever sobre a porção posterior das próteses. Processeguiu-se à moldagem com guia multifuncional durante o trans-operatório, e a carga foi feita sobre a prótese provisória com 7 dias de cicatrização, utilizando cilindros provisórios para mini-pilar. $\mathrm{O}$ tratamento foi considerado um sucesso, por parte dos profissionais e da paciente, imediatamente, prosseguindo para a etapa de confeccção da prótese definitiva após 6 meses de osseo-integração e função dos implantes.

\section{INTRODUÇÃO}

Desde a década de 80, com os estudo de P.I. Brannemark sobre a osseointegração de implantes como opção para suportar próteses totais, em substituição às convencionais, houve uma mudança no tratamento do edentulismo, altamente disseminado na população (BRÀNEMARK, 1983).

Sua inserção na área da reabilitação oral se fez rapidamente por conseguir suprir a maioria das limitações da prósete total convencional, cumprindo os mesmos parâmetros e acarretando em maior satisfação para o paciente (ABDUNABI, et al. 2019; TISCHLER, et al. 2018)

O conjunto desses fatores, junto ao aumento da expectativa de vida das populações e consequentemente do edentulismo, indicam a necessidade de maior entendimento acerca das incidências, tipos e frequência de complicações em cada modalidade de tratamento dentro do campo, e por isso estimulam a contínua descrição de casos executados (PAPASPYRIDAKOS, et al.2019; BALSHI, et al.2018; TISCHLER, et al. 2018).

Outra questão nesse tipo de reabilitação é o momento de dar carga aos implantes. A carga imediata supera muitas das desvantagens envolvidas no tratamento protético removível provisório usado durante o período de cicatrização do implante. Essas desvantagens incluem interferência na função mastigatória, fala prejudicada e possíveis efeitos adversos na estética. Além disso, a taxa de falha do implante é maior nos pacientes submetidos ao implante tratamento para uma maxila desdentada em comparação com pacientes com maxilas parcialmente desdentadas. Uma explicação é que a pressão feita sobre os tecidos moles, causada pelo uso de próteses removíveis afeta a cicatrização do implante (MIJIRITSKY, et al. 2014; HIGUCHI, LIDDELOW, 2019).

Estudos têm demonstrado altas taxas de sucesso para próteses fixas imediatas em maxilas edêntulas (ABDUNABI, et al. 2019; BALSHI, et al.2018; HIGUCHI, LIDDELOW, 2019).

Além disso, vários aspectos devem ser considerados para a carga imediata de implantes na mandíbula ou maxila edêntula, especificamente, o requisito mínimo para os tamanhos e número de implantes. Entre vários fatores encontrados para prever os resultados do tratamento 
com implantes carregados imediatamente, está uma adequada estabilidade primária (MIJIRITSKY, et al. 2014).

O trabalho em questão, busca relatar um caso de cirurgia e função imediata, maxilomandibular, incluindo o planejamento clínico, fotográfico e radiográfico e seus benefícios para a paciente.

\section{APRESENTAÇÃO DO CASO}

Paciente V.C, 60 anos, procurou o serviço de Especialização em Prótese da Associação Brasileira de Odontologia - Rio Comprido, com a queixa de que suas próteses fixas de múltiplos elementos estavam soltas e eram desconfortáveis funcional e estéticamente, comprometendo muito sua qualidade de vida. Ao primeiro exame clínico foi constatado que as próteses em questão, abrangiam 9 elementos no arco superior e 7 no inferior.

Nesse momento foram produzidos modelos de estudo e montados em articulador semiajustável. Junto disso foi feita a análise radriográfica panorâmica e observou-se um variado repertório de procedimentos à serem realizados anteriormente à reabilitação protética final, como extrações, terapia endodôntica em diversos elementos cariados, restaurações, retentores intrarradiculares, cirurgias periodontais, e coroas provisórias e definitivas.

Os objetivos do planejamento foram a correção de linha média, correção da vestibularização exagerada dos elementos superiores anteriores, melhoria estética, funcional e consequentemente da qualidade de vida.

Devido à essa soma de fatores foi considerado e apresentado à paciente o planejamento de próteses totais fixas imediatas sobre implante para ambos os arcos, com a extração de todos os elementos dentários.

Após a apresentação das inúmeras vantagens e do aceite da paciente, foi confeccionada uma guia cirúrgica após as extrações dentárias e osteotomia no modelo. Nessa etapa foi notouse grande discrepância óssea bilateral na maxila e mandíbula. No dia da cirurgia foram instalados 8 implantes no total, 4 Hexágonos Externos(HE) de 3,75X11,5mm na maxila e 4 Hexágonos Internos(HI) 4,3X11.5mm na mandíbula. Houve um approach no sentido de distalizar a saída dos implantes posteriores para diminuir o canti-lever na prótese total.

Durante a cirurgia foi feita a moldagem dos mini-pilares, sendo utilizados mini-pilares angulados de $17^{\circ}$ nos implantes mais posteriores. A moldagem foi ao laboratório para confecção da provisória e a paciente retornou em 7 dias para a captura da mesma sobre pilares provisórios sobre mini-pilares. A melhoria estética foi reconhecida imediatamente e a funcional e de qualidade de vida foram consideravelmente de acordo com o tempo de uso das próteses pela paciente e seu costume com as mesmas. Em 6 meses a paciente foi agendada para o retorno para a confecção das definitivas híbridas com resina acrílica sobre barra metálica.

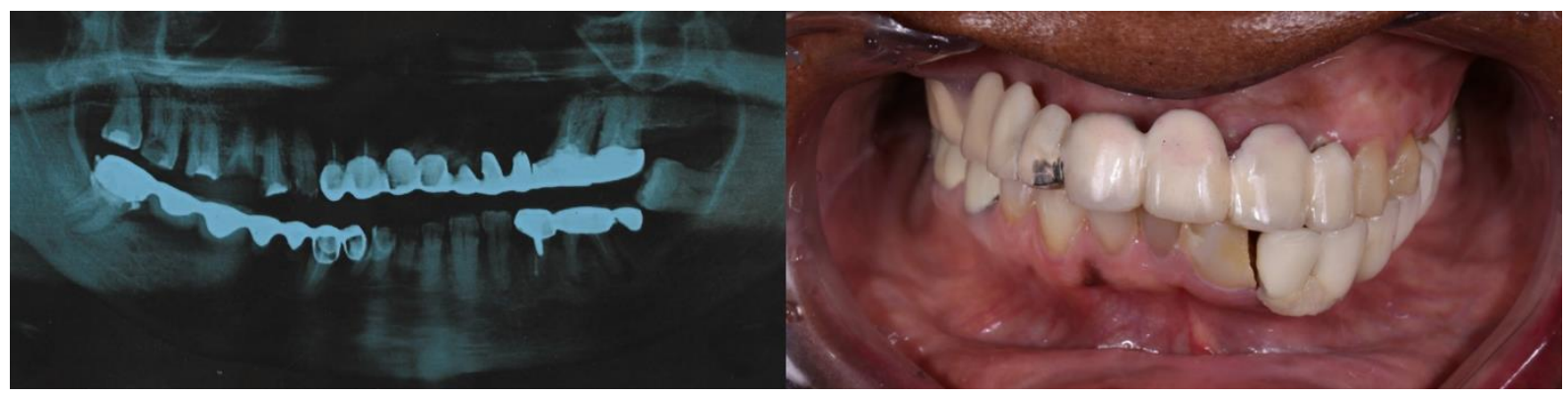

FIG.1. Radiografia Inicial e Aspecto Intraoral. 


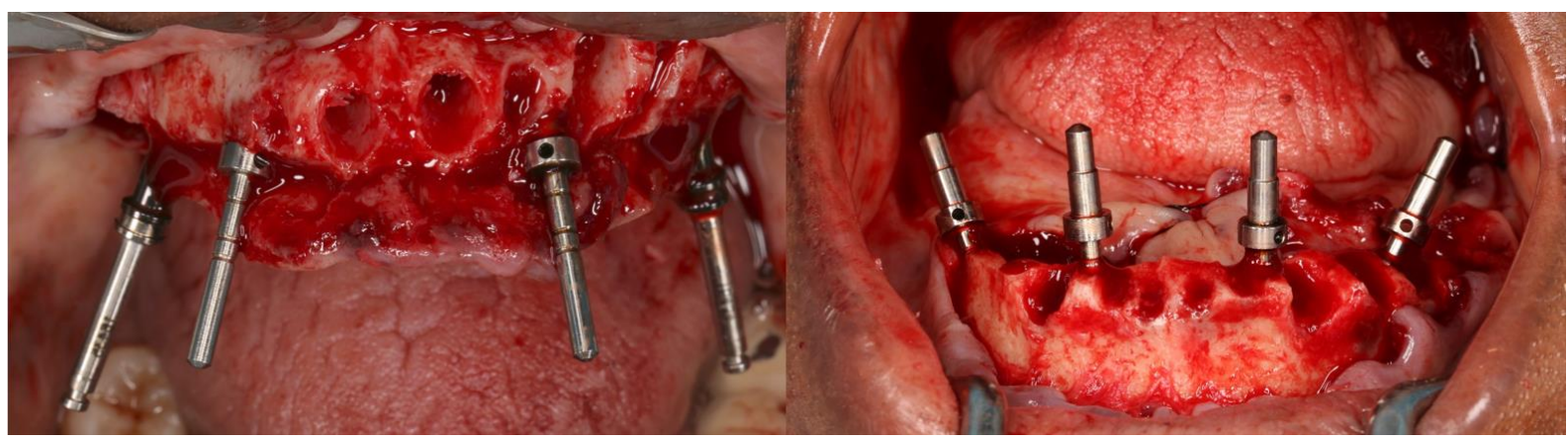

FIG.2. Posicionamento dos implantes. Inclinação distal dos implantes mais posteriores.

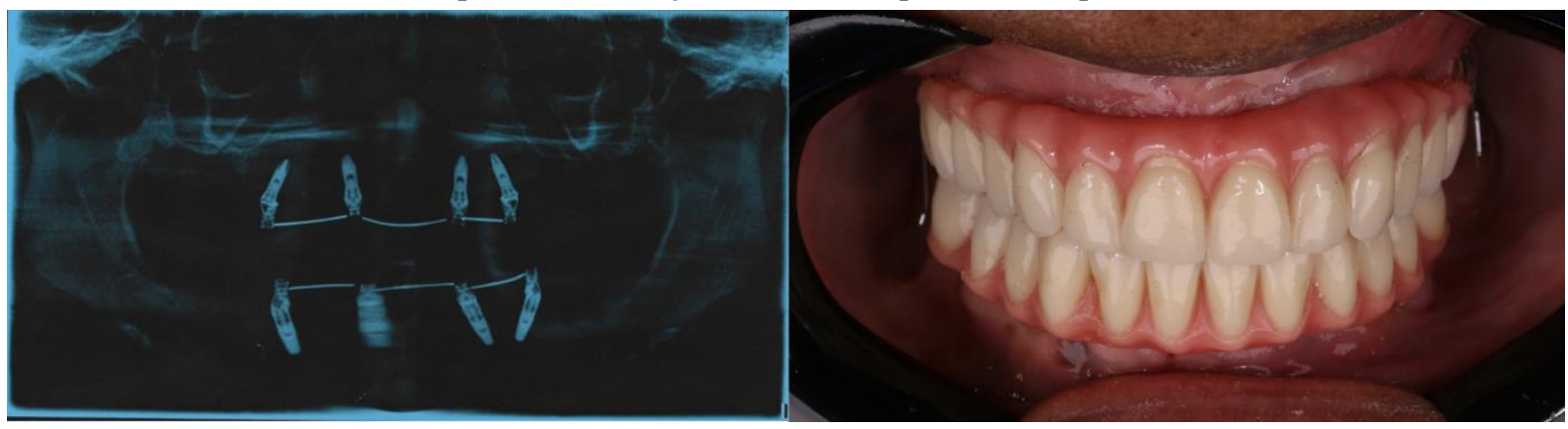

FIG.3. Radiografia Panorâmica final. Prótese provisória Imediata após captura.

\section{DISCUSSÃO}

Esse tipo de tratamento leva muito em consideração o custo benefício que trará para o paciente, o tempo de realização para sanar as queixas rapidamente e a melhoria geral da saúde. Nesse caso, foram alcançados todos os objetivos já na etapa provisória, buscando ainda alguma melhoria, especialmente nas mecânicas intra-orais no trabalho final. $\mathrm{O}$ maior questionamento foi a morbidade das extrações múltiplas e a passagem do estado dentado para o edentado do paciente em apenas um momento cirúrgico. Porém tem que se levar em consideração todos os aspectos considerados anteriormente, como o custo reduzido e o tempo ganho com esse plano de tratamento.

De acordo com a literatura, o prognóstico nos primeiros 5 anos de próteses híbridas de metal e resina, tem uma taxa de sobrevivência maior que 90\% (PAPASPYRIDAKOS, et al.2019). Das complicações técnicas menores, a mais relatada nesse período é o desgaste da prótese. Dentre as graves, a mais comum parece ser a quebra do material protético (PAPASPYRIDAKOS, et al.2019).

Sobre a imediaticidade da reabilitação, a literatura mostra que independente do momento em que recebem um tratamento protético reabiltiador fixo, os pacientes ficam mais satisfeitos do que com trabalhos removíveis. A satisfação parece varias também de acordo com o momento do tratamento em que esse quesito é questionado e de acordo com a experência pregressa do paciente com próteses totais (ABDUNABI, et al. 2019).

Quanto a estratégia para guiar o plano de tratamento, foi utilizado o conceito All-onFour, que se baseia na instalação do mínimo possível de implantes necessários para ter a mecânica funcional de suporte das cargas da mastigação sobre uma prótese fixa, com o mínimo possível de cantilever distal, maximizando a densidade óssea sem a necessidade de procedimentos de manipulação tecidual e regeneração óssea que encarecem os tratamentos e aumentam a morbidade (SOTO-PEÑALOZA, et al. 2017). Esse protocolo se baseia no posicionamento de 4 implantes na região anterior do arco em questão, para suportar uma prótese fixa imediata. Originalmente o padrão preconizado por Branemark, sugeria o posicionamento 
de 6 implantes idealmente, ou 4, sendo 2 desses o mais posterior possível e com angulações o mais paralelas entre si possível (BRANEMARK, 1983).

No relato de caso em questão, não foram testadas novas técnicas que ainda precisem de corroboração cintífica, porém o conjunto de decisões que guiaram o plano de tratamento, ou seja, conceito All-on-Four com implantes imediatos, em ambos os arcos, com carga imediata, torna o caso de alta complexidade e por isso se justifica. Em outros relatos de caso da literatura como Mijiritsky et. al., e Higuchi e Liddelow, utilizaram técnicas ainda pouco descritas na literatura e que ainda serão melhor descritas. Porém todos se baseiam na mesma bibliografia, já com grande longevidade, sobre próteses totais fixas sobre implante.

\section{CONCLUSÕES}

Após 6 meses a paciente retornou com os implantes osseointegrados e pronto para receberam a carga definitiva com a confecção da prótese híbrida com uma infraestrutura metálica passiva sob uma cobertura estética e funcional em resina acrílica. O plano de tratamento foi considerado um sucesso para a equipe e para a paciente.

\section{REFERÊNCIAS BIBLIOGRÁFICAS}

1. Branemark, P.I. Osseointegration and its experimental background. Journal of Prosthetic Dentistry. 1983;50:399-410.

2. Papaspyridakos, P.; Bordin, T. B.; Natto, Z.S. ; Kim, Y.J. ; El-Rafie, K.; Tsigarida, A.; Chochlidakis, K.; Weber, H.P. Double Full-Arch Fixed Implant-Supported Prostheses: Outcomes and Complications after a Mean Follow-Up of 5 Years. Journal of Prosthodontics. 22 February 2019.

3. Abdunabi, A.; Morris, M.; Nader, S. A.; De Souza, R. F. Impact of immediately loaded implant-supported maxillary fullarch dental prostheses: a systematic review. Journal of Applied Oral Science. 1/15 2019;27.

4. Mijiritsky, E.; Mazor, Z. ; Lorean, A.; Mortellaro, C.; Mardinger, O.; Levin, L. Transition From Hopeless Dentition to Full-Arch Fixed-Implant-Supported Rehabilitation by a Staged Extraction Approach: Rationale and Technique. The Journal of Craniofacial Surgery • Volume 25, Number 3, May 2014

5. Balshi, T. J; Wolfinger, G.J. ; Balshi, S. F. ; Bidra, A.S. A 30-Year Follow-Up of a Patient with Mandibular Complete-Arch Fixed Implant-Supported Prosthesis on 4 Implants: A Clinical Report. Journal of Prosthodontics 00 (2018) 1-6 C_ 2018 by the American College of Prosthodontists.

6. Higuchi, K.; Liddelow, G. An Innovative Implant-Supported treatment for the Edentulous Mandible: Case Report. The International Journal of Oral and Maxilofacial Implants. Quintessense Publishing. Volume 34. Number 2. 2019.

7. Tischler, M.; Patch, C.; Bidra, A.S. Rehabilitation of edentulous jaws with zirconia complete-arch fixed implant-supported prostheses: An up to 4-year retrospective clinical study. The Journal Of Prosthetic Dentistry. 2018. 
8. Soto-Peñaloza, D.; Zaragozí-Alonso, R.; Peñarrocha-Diago, M.; Peñarrocha-Diago, M. The all-on-four treatment concept: Systematic review. Journal of Clinical and Experimental Dentistry. 2017;9(3):e474-88. 